\title{
On the General Structure of Nonlinear Evolution Equations Integrable by the Two-Dimensional Matrix Spectral Problem
}

\author{
B. G. Konopelchenko
}

Institute of Nuclear Physics, 630090, Novosibirsk, USSR

\begin{abstract}
A generalization of the AKNS-technique to the two-dimensional arbitrary order matrix spectral problem is given. The general form of the integrable equations and their Bäcklund transformations in $1+2$ dimensions are found. The reduction problem is discussed.
\end{abstract}

\section{Introduction}

One of the main problems of the inverse scattering transform (IST) method is a problem of enumeration of the equations integrable by this method (see, e.g. $[1,2])$. The simple and convenient description of a class of partial differential equations integrable by the one-dimensional second-order bundle

$$
\frac{\partial \Psi}{\partial x}+\lambda A \Psi=P(x, t) \Psi
$$

has been given by Ablowitz, Kaup, Newell, and Segur (AKNS) [3]. Then this approach (AKNS-approach) has been generalized to the problem (1.0) of arbitrary order [4-9] and to some other one-dimensional spectral problems [9-11]. The infinite-dimensional group of Bäcklund transformations for these classes of integrable equations has also been found $[12,7-9]$. But up to now all the results obtained in the framework of the AKNS-approach [3-12] are concerned with equations in one spatial dimension.

The generalization of the AKNS-method to the case of several spatial dimensions is of indubitable interest. The applicability of the IST-method to the multidimensional equations has been demonstrated in $[13,14]$. Various concrete two-dimensional and multidimensional evolution equations have been considered [13-18].

Multidimensional spectral problems possess a number of specific features. Nevertheless, as we shall see, the technique described in [7-9] permits a generalization to the $1+2$ dimensions (one time and two spatial dimensions) case. 

form

In the present paper we consider a two-dimensional spectral problem of the

$$
\frac{\partial \Psi}{\partial x}+A \frac{\partial \Psi}{\partial y}=P(x, y, t) \Psi
$$

where $A$ is an arbitrary constant semisimple matrix (i.e. a diagonalizable matrix), potential $P(x, y, t)$ is a matrix $N \times N$ such that $\begin{gathered}P(x, y, t) \rightarrow 0 \\ R=\sqrt{x^{2}+y^{2}} \rightarrow \infty\end{gathered}$. The order $N$ of the matrix problem (1.1) is an arbitrary one. Spectral problem (1.1) is a natural two-dimensional generalization of the one-dimensional bundle (1.0). Spectral problems of the type (1.1) (with diagonal matrix $A$ ) and some concrete equations connected with it, have been considered earlier in [13-15, 17].

In the present paper we find the general form of nonlinear evolution equations in $1+2$ dimensions integrable by the problem (1.1). We also construct the universal infinite-dimensional group of Bäcklund transformations and infinitedimensional symmetry group for these equations. The reduction problem for general equations and some concrete reductions are considered, too. The results obtained are the generalization to the two spatial dimensions of the corresponding results for bundle (1.0) (see $[7,8]$ ). We want to note that this $1+2$ dimensional generalization is a nontrivial one and possesses various interesting features.

The paper is organized as follows. In the second section we introduce some special solutions of the linear problem (1.1), scattering matrix and obtain several important relations. In the third section we calculate the recursion operators $\hat{\Lambda}_{(n) A}, \check{\Lambda}_{(n) A}$ which play a fundamental role in our constructions. The general form of Bäcklund transformations and integrable equations are found in Sect. 4. In the fifth section the integrals of motion are calculated. The reduction problem is discussed in Sect. 6.

\section{Scattering Problem and Preliminary Relations}

We will assume that the potential $P(x, y, t) \rightarrow 0$ at $R=\sqrt{x^{2}+y^{2}} \rightarrow \infty$ so that it guarantees the existence of all integrals which will appear in our calculations and that

$$
\int_{-\infty}^{+\infty} d y \frac{\partial}{\partial y}(\ldots)=0
$$

We will also assume that the potential satisfies the gauge condition $P_{0} \equiv 0$, where $P_{0}$ is a projection of potential $P$ onto $g_{0}$-component of the Fitting decomposition with respect to $A$. Let us recall shortly its properties (see, e.g. [19]). Fitting decomposition of the general linear matrix algebra $\mathrm{gl}(N, C)$ with respect to a semisimple matrix $A$ is a decomposition into the tensor sum $\operatorname{gl}(N, C)$ $=g_{0} \oplus g_{F}$, where $g_{0}$ is a subalgebra of matrices commuting with $A$ $\left(g_{0}=\{g \in \operatorname{gl}(N, C),[g, A]=0\}\right)$ and $g_{F}$ is a tensor sum of nonzero root subspaces. Then $\left[g_{0}, g_{0}\right] \subset g_{0}$ and $\left[g_{0}, g_{F}\right] \subset g_{F}$. For an arbitrary matrix $B$ of the order $N$ we have a simple decomposition $B=B_{0}+B_{F}$, where $B_{0}$ is a projection of $B$ onto $g_{0}$ and $B_{F}$ is a projection of $B$ onto $g_{F}$. We have the decomposition $P(x, y, t)$ $=P_{0}(x, y, t)+P_{F}(x, y, t)$, too. Using the invariance of the problem (1.1) under the 
transformations $\Psi \rightarrow \Psi^{\prime}=G(x, y) \Psi, \quad P \rightarrow P^{\prime}=G P G^{-1}+\left(\frac{\partial G}{\partial x}+A \frac{\partial G}{\partial y}\right) G^{-1}$, where $G=G_{0}$, it is always possible to choose $P_{0}=0$. A meaning of the gauge $P_{0}=0$ consists in the excluding of pure gauge (nondynamical) degrees of freedom from $P(x, y, t)$.

Let us now consider the linear problem (1.1). We will denote the solutions of this problem by $\hat{\Psi}$. Following [15], we introduce matrices-solutions $\hat{F}_{\lambda}^{+}(x, y)$ and $\hat{F}_{\lambda}^{-}(x, y)$ of the problem (1.1) given by their asymptotic behaviour

$$
\hat{F}_{\lambda}^{+}(x, y) \underset{x \rightarrow+\infty}{\longrightarrow}(2 \pi i)^{-1 / 2} e^{\lambda(y-A x)}, \quad \hat{F}_{\lambda}^{-}(x, y) \underset{x \rightarrow-\infty}{\longrightarrow}(2 \pi i)^{-1 / 2} e^{\lambda(y-A x)},
$$

and the scattering matrix $\hat{S}(\tilde{\lambda}, \lambda, t)$ :

$$
\hat{F}_{\lambda}^{+}(x, y, t)=\int_{-i \infty}^{+i \infty} d \tilde{\lambda} \hat{F}_{\tilde{\lambda}}^{-}(x, y, t) \hat{S}(\tilde{\lambda}, \lambda, t) .
$$

Let us consider also the problem adjoint to (1.1):

$$
\frac{\partial \check{\Psi}}{\partial x}+\frac{\partial \check{\Psi}}{\partial y} A=-\check{\Psi} P(x, y, t)
$$

We introduce the matrices-solutions $\check{F}_{\lambda}^{+}(x, y)$ and $\check{F}_{\lambda}^{-}(x, y)$ of the problem (2.1)

$$
\check{F}_{\lambda}^{+}(x, y) \underset{x \rightarrow+\infty}{\longrightarrow}(2 \pi i)^{-1 / 2} e^{-\lambda(y-A x)}, \quad \check{F}_{\lambda}^{-}(x, y) \underset{x \rightarrow-\infty}{\longrightarrow}(2 \pi i)^{-1 / 2} e^{-\lambda(y-A x)},
$$

and the corresponding scattering matrix $\check{S}(\lambda, \tilde{\lambda}, t)$ :

$$
\check{F}_{\lambda}^{+}(x, y, t)=\int_{-i \infty}^{+i \infty} d \tilde{\lambda} \check{S}(\lambda, \tilde{\lambda}, t) \check{F}_{\tilde{\lambda}}^{-}(x, y, t) .
$$

Let us note that in contrast with the one-dimensional problem (1.0), the matrix $\hat{\Psi}^{-1}$ is not a solution of the adjoint problem (2.1). It is easy to show that the following relations hold:

$$
\begin{array}{r}
\int_{-\infty}^{+\infty} d y \breve{F}_{\tilde{\lambda}}^{ \pm}(x, y, t) \hat{F}_{\lambda}^{ \pm}(x, y, t)=\delta(\tilde{\lambda}-\lambda), \\
\int_{-i \infty}^{+i \infty} d \lambda \hat{F}_{\lambda}^{ \pm}(x, y, t) \breve{F}_{\lambda}^{ \pm}\left(x, y^{\prime}, t\right)=\delta\left(y-y^{\prime}\right), \\
\int_{-i \infty}^{+i \infty} d \mu \check{S}(\tilde{\lambda}, \mu, t) \hat{S}(\mu, \lambda, t)=\delta(\tilde{\lambda}-\lambda),
\end{array}
$$

where $\delta(\lambda)$ is a Dirac delta-function.

By virtue of $(2.2)$, the scattering matrices $\hat{S}(\tilde{\lambda}, \lambda, t)$ and $\check{S}(\tilde{\lambda}, \lambda, t)$ can be represented as follows

$$
\begin{aligned}
& \hat{S}(\tilde{\lambda}, \lambda, t)=\int_{-\infty}^{+\infty} d y \check{F}_{\tilde{\lambda}}^{-}(x, y, t) \hat{F}_{\lambda}^{+}(x, y, t), \\
& \check{S}(\tilde{\lambda}, \lambda, t)=\int_{-\infty}^{+\infty} d y \check{F}_{\tilde{\lambda}}^{+}(x, y, t) \hat{F}_{\lambda}^{-}(x, y, t) .
\end{aligned}
$$


Now let $P$ and $P^{\prime}$ be two different potentials and $\hat{\Psi}, \hat{\Psi}^{\prime}, \check{\Psi}^{\prime}, \breve{\Psi}^{\prime}$ are the corresponding solutions of the problems (1.1) and (2.1). Using (1.1), (2.1) and taking into account (2.3), one can show that

$$
\begin{aligned}
& \hat{\Psi}_{\lambda}^{\prime}(x, y)-\int_{-i \infty}^{+i \infty} d \tilde{\lambda} \hat{\Psi}_{\tilde{\lambda}}(x, y) K(\tilde{\lambda}, \lambda) \\
& =-\int_{-i \infty}^{+i \infty} d \tilde{\lambda} \hat{\Psi}_{\tilde{\lambda}}(x, y) \int_{x}^{\infty} d z \int_{-\infty}^{+\infty} d y^{\prime} \breve{\Psi}_{\tilde{\lambda}}\left(z, y^{\prime}\right)\left(P^{\prime}\left(z, y^{\prime}\right)-P\left(z, y^{\prime}\right)\right) \hat{\Psi}_{\lambda}^{\prime}\left(z, y^{\prime}\right),
\end{aligned}
$$

where $K(\tilde{\lambda}, \lambda)=\left.\int_{-\infty}^{+\infty} d y \check{\Psi}_{\tilde{\lambda}}(x, y) \hat{\Psi}_{\lambda}^{\prime}(x, y)\right|_{x=+\infty}$. Putting $\Psi_{\lambda}=\hat{F}_{\lambda}^{+}$in $(2.5)$ and proceeding to the limit $x \rightarrow-\infty$, one gets

$$
\begin{aligned}
& \hat{S}^{\prime}(\tilde{\lambda}, \lambda)-\hat{S}(\tilde{\lambda}, \lambda) \\
& \quad=-\int_{-i \infty}^{+i \infty} d \mu \hat{S}(\tilde{\lambda}, \mu) \int_{-\infty}^{+\infty} d x d y \check{F}_{\mu}^{+}(x, y)\left(P^{\prime}(x, y)-P(x, y)\right) \hat{F}_{\lambda}^{+\prime}(x, y) .
\end{aligned}
$$

Formula (2.6) which connects a variation of the potential $P$ to that of the scattering matrix plays a fundamental role in further considerations. Putting $P=0$ we obtain from (2.6) the following relation between the potential $P(x, y, t)$ and the scattering matrix $\hat{S}(\tilde{\lambda}, \lambda, t)$ :

$$
\hat{S}(\tilde{\lambda}, \lambda, t)=\delta(\tilde{\lambda}-\lambda)-(2 \pi i)^{-1 / 2} \int_{-\infty}^{+\infty} d x d y e^{-\tilde{\lambda}(y-A x)} P(x, y, t) \hat{F}_{\lambda}^{+}(x, y, t) .
$$

In particular, for small (in a suitable sense) potential $P(x, y, t)$ we have in the linear approximation

$$
\hat{S}(\tilde{\lambda}, \lambda, t)=\delta(\tilde{\lambda}-\lambda)-(2 \pi i)^{-1} \int_{-\infty}^{+\infty} d x d y e^{(\lambda-\tilde{\lambda}) y} e^{\tilde{\lambda} A x} P(x, y, t) e^{-\lambda A x} .
$$

The mapping $P(x, y, t) \rightarrow S(\tilde{\lambda}, \lambda, t)$ given by the spectral problem (1.1) determines a correspondence between the transformations $P \rightarrow P^{\prime}$ on the manifold of potentials $\{P(x, y, t)\}$ and the transformations $S \rightarrow S^{\prime}$ on the manifold of scattering matrices $\{S(\tilde{\lambda}, \lambda, t)\}$. This fact follows from the commutative diagram

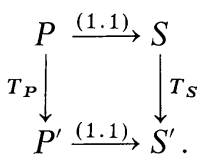

Let us now consider only the transformations $T$ such that

$$
\hat{S}(\tilde{\lambda}, \lambda, t) \stackrel{T_{s}}{\longrightarrow} \hat{S}^{\prime}(\tilde{\lambda}, \lambda, t)=B^{-1}(\tilde{\lambda}, t) \hat{S}(\tilde{\lambda}, \lambda, t) C(\lambda, t),
$$

where $B(\tilde{\lambda}, t)$ and $C(\lambda, t)$ are (in general, arbitrary) matrices commuting with $A$, i.e. $B=B_{0}, C=C_{0}$. The "restricted" transformations of the type (2.7) are, as we shall see, wide enough. 
Combining the relation (2.6) with (2.7) and taking into account (2.4), one finds

$$
\begin{array}{r}
\int_{-i \infty}^{+i \infty} d \mu \check{S}(\tilde{\lambda}, \mu)(1-B(\mu, t)) \hat{S}^{\prime}(\mu, \lambda)-(1-C(\lambda, t)) \delta(\tilde{\lambda}-\lambda) \\
=-\int_{-\infty}^{+\infty} d x d y \check{F}_{\tilde{\lambda}}^{+}(x, y)\left(P^{\prime}(x, y)-P(x, y)\right) \hat{F}_{\lambda}^{+{ }^{\prime}}(x, y) .
\end{array}
$$

Then one can prove the following identity:

$$
\begin{aligned}
& \int_{-i \infty}^{+i \infty} d \mu \check{S}(\tilde{\lambda}, \mu)(1-B(\mu, t)) \hat{S}^{\prime}(\mu, \lambda)-\delta(\tilde{\lambda}-\lambda)(1-B(\lambda, t)) \\
&=-\left.\int_{-\infty}^{+\infty} d y \check{F}_{\tilde{\lambda}}^{+}(x, y)\left(1-B\left(\frac{\partial}{\partial y}, t\right)\right) \hat{F}_{\lambda}^{+{ }^{\prime}}(x, y)\right|_{x=-\infty} ^{x=+\infty} \\
&= \int_{-\infty}^{+\infty} d x d y \check{F}_{\tilde{\lambda}}^{+}(x, y)\left(P(x, y)\left(1-B\left(\frac{\partial}{\partial y}, t\right)\right) \hat{F}_{\lambda}^{+{ }^{\prime}}(x, y)\right. \\
&\left.-\left(1-B\left(\frac{\partial}{\partial y}, t\right)\right) P^{\prime}(x, y) \hat{F}_{\lambda}^{+\prime}(x, y)\right) .
\end{aligned}
$$

Taking the projection of the equality (2.8) onto $g_{F}$ and using the identity (2.9), we obtain

$$
\begin{gathered}
\int_{-\infty}^{+\infty} d x d y\left\{\check{F}_{\tilde{\lambda}}^{+}(x, y)\left(B\left(\frac{\partial}{\partial y}, t\right)\right) P^{\prime}(x, y) \hat{F}_{\lambda}^{+\prime}(x, y)\right. \\
\left.-P(x, y) B\left(\frac{\partial}{\partial y}, t\right) \hat{F}_{\lambda}^{+\prime}(x, y)\right\}_{F}=0 .
\end{gathered}
$$

The matrix $B\left(\frac{\partial}{\partial y}, t\right)$ which is contained in formulae (2.10) can be represented in the form $B\left(\frac{\partial}{\partial y}, t\right)=\sum_{\alpha=1}^{r_{A}} B_{\alpha}\left(\frac{\partial}{\partial y}, t\right) H_{\alpha}$, where matrices $H_{\alpha}\left(\alpha=1, \ldots, r_{A}\right)$ form a basis of the subalgebra $g_{0}$ and $B_{\alpha}\left(\frac{\partial}{\partial y}, t\right)$ are functions. Below we will consider only the entire functions $B_{\alpha}\left(\frac{\partial}{\partial y}, t\right)$, i.e. $B_{\alpha}\left(\frac{\partial}{\partial y}, t\right)=\sum_{n=0}^{\infty} b_{\alpha n}(t)\left(\frac{\partial}{\partial y}\right)^{n}$, where $b_{\alpha n}(t)$ are arbitrary functions. For such functions $B_{\alpha}\left(\frac{\partial}{\partial y}, t\right)$, the equality (2.10) can be rewritten as
follows

$$
\begin{aligned}
& \int_{-\infty}^{+\infty} d x d y \sum_{\alpha=1}^{r_{A}} \sum_{n=0}^{\infty} b_{\alpha n}(t) \operatorname{tr}\left(H_{\alpha} P^{\prime}(x, y)(-1)^{n} \breve{\Phi}_{(n) F}^{(F)}\right. \\
& \left.-P(x, y) H_{\alpha} \hat{\Phi}_{(n) F}^{(F)}\right)=0,
\end{aligned}
$$

where tr is a usual matrix trace and

$$
\begin{aligned}
& \left(\hat{\Phi}_{(n)}^{(i m)}\right)_{k l} \stackrel{\text { def }}{=} \frac{\partial^{n}\left(\hat{F}_{\lambda}^{+}\right)_{k m}^{\prime}}{\partial y^{n}}\left(\check{F}_{\tilde{\lambda}}^{+}\right)_{i l}, \\
& \left(\check{\Phi}_{(n)}^{(i m)}\right)_{k l} \stackrel{\text { def }}{=}\left(\hat{F}_{\lambda}^{+}\right)_{k m}^{\prime} \frac{\partial^{n}\left(\check{F}_{\tilde{\lambda}}^{+}\right)_{i l}}{\partial y^{n}} \quad(n=0,1,2, \ldots) .
\end{aligned}
$$




\section{The Recursion Operators}

For further transformations of the equality (2.11) one must establish the relations between the expressions $\Phi_{(n) F}^{(F)}$ with different $n$.

Let us first consider the expression $\hat{\Phi}_{(n) F}$. Let us differentiate $n-1$ times over variable $y$ the system (1.1) for $\left(\hat{F}_{\lambda}^{+}\right)_{k m}^{\prime}$ and then multiply the obtained equation by $\breve{F}_{i l}^{+}$. Forming in the obtained equality the total derivatives over $x$ and $y$ and taking into account (2.1), one gets the equation

$$
\begin{aligned}
& \frac{\partial \hat{\Phi}_{(n-1)}}{\partial x}+\frac{\partial \hat{\Phi}_{(n-1)}}{\partial y} A=-\left[A, \hat{\Phi}_{(n)}\right] \\
& \quad+P^{\prime} \hat{\Phi}_{(n-1)}-\hat{\Phi}_{(n-1)} P+\sum_{m=0}^{n-2} C_{m}^{n-1} P_{(n-1-m)}^{\prime} \hat{\Phi}_{(m)},
\end{aligned}
$$

where $C_{m}^{n}=\frac{n !}{m !(n-m) !}$ and $P_{(k)} \stackrel{\text { def }}{=} \frac{\partial^{k} P(x, y)}{\partial y^{k}}$. Then if one projects the Eq. (3.1) onto $g_{0}$ and uses the relation $\left(\Phi \Psi_{F}\right)_{0}=\left(\Phi_{F} \Psi_{F}\right)_{0}$, one gets

$$
\begin{aligned}
& \frac{\partial \hat{\Phi}_{(n-1) 0}}{\partial x}+\frac{\partial \hat{\Phi}_{(n-1) 0}}{\partial y} A \\
& \quad=\left(P^{\prime} \hat{\Phi}_{(n-1) F}-\hat{\Phi}_{(n-1) F} P+\sum_{m=0}^{n-2} C_{m}^{n-1} P_{(n-1-m)}^{\prime} \hat{\Phi}_{(m) F}\right)_{0} .
\end{aligned}
$$

The integration of (3.2) gives

$$
\begin{aligned}
\hat{\Phi}_{(n-1) 0}(x, y)= & \hat{\Phi}_{(n-1) 0}(x=+\infty, y) \\
& -\mathscr{I}^{-}\left(P^{\prime} \hat{\Phi}_{(n-1) F}-\hat{\Phi}_{(n-1) F} P+\sum_{m=0}^{n-2} C_{m}^{n-1} P_{(n-1-m)}^{\prime} \hat{\Phi}_{(m) F}\right)_{0},
\end{aligned}
$$

where for $f_{0}(x, y)=\sum_{\alpha=1}^{r_{A}} f_{\alpha}(x, y) H_{\alpha}$ and $A=\sum_{\alpha=1}^{r_{A}} a_{\alpha} H_{\alpha}$,

$$
\left(\mathscr{I}^{-} f_{0}\right)(x, y) \stackrel{\text { def }}{=} \sum_{\alpha=1}^{r_{A}} H_{\alpha} \int_{x}^{\infty} d z f_{\alpha}\left(z, a_{\alpha}(z-x)+y\right) .
$$

So one can express the quantities $\Phi_{(n) 0}$ through the quantities $\hat{\Phi}_{(m) F}$ $(m=0,1, \ldots, n-1)$. As a result taking the projection of the Eq. (3.1) onto subspace $g_{F}$, taking into account the equality (3.3) and the relations $\left(\Phi_{0} \Psi_{F}\right)_{F}=\Phi_{0} \Psi_{F}$, $\hat{\Phi}_{(n) 0}^{F}(x=+\infty, y)=0$, we obtain

$$
\begin{aligned}
{\left[A, \hat{\Phi}_{(n) F}^{(F)}\right]=} & \hat{\Lambda}_{(1)} \hat{\Phi}_{(n-1) F}^{(F)} \\
& +\sum_{m=0}^{n-2} C_{m}^{n-1}\left\{\left(P_{(n-1-m)}^{\prime} \hat{\Phi}_{(m) F}^{(F)}\right)_{F}-P^{\prime} \mathscr{I}^{-}\left(P_{(n-1-m)}^{\prime} \hat{\Phi}_{(m) F}^{(F)}\right)_{0}\right. \\
& +\mathscr{I}^{-}\left(P_{(n-1-m)}^{\prime} \hat{\Phi}_{(m) F}^{(F)}\right)_{0} \cdot P-P_{(n-1-m)}^{\prime} \mathscr{I}^{-}\left(P^{\prime} \hat{\Phi}_{(m) F}^{(F)}-\hat{\Phi}_{(m) F}^{(F)} P\right)_{0} \\
& \left.-\sum_{l=0}^{m-1} C_{l}^{m} P_{(n-1-m)}^{\prime} \mathscr{I}^{-}\left(P_{(m-l)}^{\prime} \hat{\Phi}_{(l) F}^{(F)}\right)_{0}\right\}, \quad(n=1,2,3, \ldots),
\end{aligned}
$$


where operator $\hat{\Lambda}_{(1)}$ acts as follows

$$
\begin{aligned}
\hat{\Lambda}_{(1)} \Phi= & -\frac{\partial \Phi}{\partial x}-\frac{\partial \Phi}{\partial y} A+\left(P^{\prime} \Phi-\Phi P\right)_{F} \\
& -P^{\prime} \mathscr{I}^{-}\left(P^{\prime} \Phi-\Phi P\right)_{0}+\mathscr{I}^{-}\left(P^{\prime} \Phi-\Phi P\right)_{0} \cdot P .
\end{aligned}
$$

From the relations (3.4) it follows that there exist such operators $\hat{\Lambda}_{(n) A}$ that

$$
\hat{\Phi}_{(n) F}^{(F)}=\Lambda_{(n) A} \Phi_{(0) F}^{(F)} \quad(n=1,2,3, \ldots),
$$

where $\hat{\Lambda}_{(n) A} \Phi \stackrel{\text { def }}{=}\left(\hat{\Lambda}_{(n)} \Phi\right)_{A},\left[A, \Phi_{A}\right] \stackrel{\text { def }}{=} \Phi$ and $\left(\Phi_{(0)}^{(i m)}\right)_{k l} \stackrel{\text { def }}{=}\left(\hat{F}^{+}\right)_{k m}^{\prime}\left(\check{F}^{+}\right)_{i l}$. The operators $\hat{\Lambda}_{(n) A}$ are determined by the following recursion relations:

$$
\begin{aligned}
& \hat{\Lambda}_{(n) A} \Phi=\hat{\Lambda}_{(1) A} \hat{\Lambda}_{(n-1) A} \Phi \\
& \quad+\sum_{m=0}^{n-2} C_{m}^{n-1}\left\{\left(P_{(n-1-m)}^{\prime} \hat{\Lambda}_{(m) A} \Phi\right)_{F}-P^{\prime} \mathscr{I}^{-}\left(P_{(n-1-m)}^{\prime} \hat{\Lambda}_{(m) A} \Phi\right)_{0}\right. \\
& \quad+\mathscr{I}^{-}\left(P_{(n-1-m)}^{\prime} \hat{\Lambda}_{(m) A} \Phi\right)_{0} \cdot P-P_{(n-1-m)}^{\prime} \mathscr{I}^{-}\left(P^{\prime} \hat{\Lambda}_{(m) A} \Phi\right. \\
& \left.\left.\quad-\hat{\Lambda}_{(m) A} \Phi \cdot P\right)_{0}-\sum_{l=0}^{m-1} C_{l}^{m} P_{(n-1-m)}^{\prime} \mathscr{I}^{-}\left(P_{(m-l)}^{\prime} \hat{\Lambda}_{(l) A} \Phi_{0}\right)\right\}_{A} \quad(n=2,3, \ldots) .
\end{aligned}
$$

In a similar way one shows that

$$
\check{\Phi}_{(n) F}^{(F)}=\check{\Lambda}_{(n) A} \Phi_{(0) F}^{(F)} \quad(n=1,2,3, \ldots) .
$$

Operators $\check{\Lambda}_{(n) A}$ are determined by the recursion relations

$$
\begin{aligned}
\check{\Lambda}_{(n) A} \Phi= & \check{\Lambda}_{(1) A} \check{\Lambda}_{(n-1) A} \Phi \\
& +\sum_{m=0}^{n-2} C_{m}^{n-1}\left\{\left(\check{\Lambda}_{(m) A} \Phi \cdot P_{(n-1-m)}\right)_{F}-P^{\prime} \mathscr{I}^{-}\left(\check{\Lambda}_{(m) A} \Phi \cdot P_{(n-1-m)}\right)_{0}\right. \\
& +\mathscr{I}^{-}\left(\check{\Lambda}_{(m) A} \Phi \cdot P_{(n-1-m)}\right)_{0} \cdot P-\mathscr{I}^{-}\left(P^{\prime} \check{\Lambda}_{(m) A} \Phi-\check{\Lambda}_{(m) A} \Phi \cdot P\right)_{0} \\
& \left.\cdot P_{(n-1-m)}+\sum_{l=0}^{m-1} C_{l}^{m} \mathscr{I}^{-}\left(\check{\Lambda}_{(l) A} \Phi \cdot P_{(m-l)}\right)_{0} \cdot P_{(n-1-m)}\right\}_{A} \quad(n=2,3, \ldots),
\end{aligned}
$$

where

$$
\begin{aligned}
\check{\Lambda}_{(1)} \Phi= & \frac{\partial \Phi}{\partial x}+A \frac{\partial \Phi}{\partial y}-\left(P^{\prime} \Phi-\Phi P\right)_{F} \\
& +P^{\prime} \mathscr{I}^{-}\left(P^{\prime} \Phi-\Phi P\right)_{0}-\mathscr{I}^{-}\left(P^{\prime} \Phi-\Phi P\right)_{0} \cdot P .
\end{aligned}
$$

The operators $\hat{\Lambda}_{(n) A}$ and $\check{\Lambda}_{(n) A}$ are not independent. Throwing the derivative $\frac{\partial}{\partial y}$ from $\check{F}^{+}$to $\hat{F}^{+\prime}$, for example in the expression $\check{\Phi}_{(n)}$, it is not difficult to show that

$$
\check{\Lambda}_{(n) A}=\sum_{k=0}^{n}(-1)^{k} C_{k}^{n} \frac{\partial^{n-k}}{\partial y^{n-k}} \hat{\Lambda}_{(k) A} \quad(n=1,2, \ldots) .
$$


In the further constructions we will use also the operators $\hat{\Lambda}_{(n) A}^{+}, \check{\Lambda}_{(n) A}^{+}$adjoint to the operators $\hat{\Lambda}_{(n) A}, \breve{\Lambda}_{(n) A}$ with respect to the bilinear form

$$
\langle\chi, \Psi\rangle=\int_{-\infty}^{+\infty} d x d y \operatorname{tr}\left(\chi_{F}(x, y) \Psi_{F}(x, y)\right)
$$

The corresponding recursion relations, for example, for operators $\hat{\Lambda}_{(n) A}^{+}$are of the form

$$
\begin{aligned}
& \hat{\Lambda}_{(n) A}^{+} \Phi=\hat{\Lambda}_{(n-1) A}^{+} \hat{\Lambda}_{(1) A}^{+} \Phi-\sum_{m=0}^{n-2} C_{m}^{n-1} \hat{\Lambda}_{(m) A}^{+}\left\{\left(\Phi_{A} P_{(n-1-m)}^{\prime}\right)_{F}\right. \\
& -\mathscr{I}^{+}\left(\Phi_{A} P^{\prime}-P \Phi_{A}\right)_{0} \cdot P_{(n-1-m)}^{\prime}-\mathscr{I}^{+}\left(\Phi_{A} P_{(n-1-m)}^{\prime}\right) \cdot P^{\prime} \\
& \left.+P \cdot \mathscr{I}^{+}\left(\Phi_{A} P_{(n-1-m)}^{\prime}\right)_{0}\right\}+\sum_{m=0}^{n-2} C_{m}^{n-1} \sum_{l=0}^{m-1} C_{l}^{m} \hat{\Lambda}_{(l) A}^{+} \cdot \mathscr{I}^{+}\left(\Phi_{A} P_{(n-1-m)}^{\prime}\right)_{0} \cdot P_{(m-l)}^{\prime},
\end{aligned}
$$

where $\hat{\Lambda}_{(n) A}^{+} \Phi=-\hat{\Lambda}_{(n)}^{+} \Phi_{A}$ and

$$
\begin{aligned}
\hat{\Lambda}_{(1)}^{+} \Phi= & \frac{\partial \Phi}{\partial x}+A \frac{\partial \Phi}{\partial y}+\left(\Phi P^{\prime}-P \Phi\right)_{F} \\
& +P \mathscr{I}^{+}\left(\Phi P^{\prime}-P \Phi\right)_{0}-\mathscr{I}^{+}\left(\Phi P^{\prime}-P \Phi\right)_{0} \cdot P^{\prime},
\end{aligned}
$$

where

$$
\left(\mathscr{I}^{+} f_{0}\right)(x, y) \stackrel{\operatorname{def}}{=} \sum_{\alpha=1}^{r_{A}} H_{\alpha} \int_{-\infty}^{x} d z f_{\alpha}\left(z, a_{\alpha}(z-x)+y\right) .
$$

The operators $\breve{\Lambda}_{(n) A}^{+}$can be determined from the recursion relations analogous to (3.12) or from the relations

$$
\check{\Lambda}_{(n) A}^{+}=(-1)^{n} \sum_{k=0}^{n} C_{k}^{n} \hat{\Lambda}_{(k) A}^{+} \frac{\partial^{n-k}}{\partial y^{n-k}} .
$$

Analogously to (3.11) and (3.14) one can express the operators $\hat{\Lambda}_{(n) A}$ and $\hat{\Lambda}_{(n) A}^{+}$ through respectively $\check{\Lambda}_{(m) A}$ and $\check{\Lambda}_{(m) A}^{+}$.

\section{General Structure of the Integrable Equations and Bäcklund-Transformations}

The existence of the recursion operators of the type $\Lambda_{(n) A}$ and $\Lambda_{(n) A}^{+}$is extremely important in the generalized AKNS-method. In our case the relations (3.6) and (3.8) allow us to rewrite the equality (2.11) in the form

$$
\begin{aligned}
& \int_{-\infty}^{+\infty} d x d y \sum_{\alpha=1}^{r_{A}} \sum_{n=0}^{\infty} b_{\alpha n}(t) \operatorname{tr}\left(H_{\alpha} P^{\prime}(x . y)(-1)^{n} \check{\Lambda}_{(n) A} \Phi_{(0) F}^{(F)}\right. \\
& \left.\quad-P(x, y) H_{\alpha} \hat{\Lambda}_{(n) A} \Phi_{(0) F}^{(F)}\right)=0,
\end{aligned}
$$

where $\hat{\Lambda}_{(0)}=\check{\Lambda}_{(0)} \equiv 1$. 
From (4.1) we have

$$
\begin{array}{r}
\int_{-\infty}^{+\infty} d x d y \operatorname{tr}\left(\Phi _ { ( 0 ) F } ^ { ( F ) } ( x , y ) \cdot \left\{\sum_{\alpha=1}^{r_{A}} \sum_{n=0}^{\infty} b_{\alpha n}(t)\right.\right. \\
\left.\left.\cdot\left((-1)^{n} \check{\Lambda}_{(n) A}^{+} H_{\alpha} P^{\prime}-\hat{\Lambda}_{(n) A}^{+} P H_{\alpha}\right)\right\}\right)=0,
\end{array}
$$

where operators $\hat{\Lambda}_{(n) A}^{+}$and $\check{\Lambda}_{(n) A}^{+}$are given by formulas (3.12)-(3.14).

The equality (4.2) is fulfilled if

$$
\sum_{\alpha=1}^{r_{A}}\left(B_{\alpha}\left(\check{\Lambda}_{A}^{+}, t\right) H_{\alpha} P^{\prime}-B_{\alpha}\left(\hat{\Lambda}_{A}^{+}, t\right) P H_{\alpha}\right)=0
$$

where

$$
\begin{aligned}
& B_{\alpha}\left(\check{\Lambda}_{A}^{+}, t\right) \stackrel{\operatorname{def}}{=} \sum_{n=0}^{\infty}(-1)^{n} b_{\alpha n}(t) \check{\Lambda}_{(n) A}^{+}, \\
& B_{\alpha}\left(\hat{\Lambda}_{A}^{+}, t\right) \stackrel{\operatorname{def}}{=} \sum_{n=0}^{\infty} b_{\alpha n}(t) \hat{\Lambda}_{(n) A}^{+} .
\end{aligned}
$$

If $\Phi_{(0) F}^{(F)}(x, y)$ form a complete set (similar to the one-dimensional case) then Eq. (4.3) is also a necessary condition of the fulfillment of the equality (4.2).

The relation (4.3) just determines the transformation of the potential $P \rightarrow P^{\prime}$, which corresponds to the transformation of the scattering matrix $S \rightarrow S^{\prime}$ of the form (2.7). It is important that the relation (4.3) contains only the potential $P$ and the transformed potential $P^{\prime}$. We restricted ourselves by the transformation law (2.7) in order to convert the transformation law of the scattering matrix into the explicit transformation law of the potential which contains only $P$ and $P^{\prime}$.

The transformations (2.7), (4.3) form, as it is easy to see from (2.7), an infinitedimensional group. If $A$ is regular matrix (i.e. all eigenvalues of $A$ are different), then subalgebra $g_{0}$ is abelian. In this case the group of transformations $(2.7),(4.3)$ is an infinite-dimensional abelian group.

The structure of this group of transformations (2.7), (4.3) (Group B) is determined by the spectral problem (1.1). Group $B$ which acts on the manifold of the potentials $\{P(x, y, t)\}$ by the formula (4.3) and on the manifold of the scattering matrices $\{S(\tilde{\lambda}, \lambda, t)\}$ by the formula (2.7), plays a fundamental role in the analysis of nonlinear systems connected with the problem (1.1) and their properties.

Let us consider a one-parameter subgroup of this group given by

$$
B=C=\sum_{\alpha=1}^{r_{A}} \exp \left\{-\int_{t}^{t^{\prime}} d s \Omega_{\alpha}(\lambda, s)\right\} H_{\alpha},
$$

where $\Omega_{\alpha}(\lambda, t)$ are some functions entire on $\lambda\left(\Omega_{\alpha}(\lambda, t)=\sum_{n=0}^{\infty} \omega_{\alpha n}(t) \cdot \lambda^{n}\right)$.

It is not difficult to see that the transformation (2.7) with matrices $B$ and $C$ of the form (4.4) is a displacement in time $t$ :

$$
\hat{S}(\tilde{\lambda}, \lambda, t) \rightarrow \hat{S}^{\prime}(\tilde{\lambda}, \lambda, t)=B^{-1} \hat{S}(\tilde{\lambda}, \lambda, t) B=\hat{S}\left(\tilde{\lambda}, \lambda, t^{\prime}\right) .
$$


The corresponding transformation of the potential is $P(x, y, t) \rightarrow P^{\prime}(x, y, t)$ $=P\left(x, y, t^{\prime}\right)$ and is given by the formula ${ }^{1}$

$$
\begin{array}{r}
\sum_{\alpha=1}^{r_{A}} \sum_{n=0}^{\infty}\left\{\exp \left(-\int_{t}^{t^{\prime}} d s \omega_{\alpha n}(S) \check{\Lambda}_{(n) A}^{+}\right)(-1)^{n} H_{\alpha} P\left(x, t^{\prime}\right)\right. \\
\left.-\exp \left(-\int_{t}^{t^{\prime}} d s \omega_{\alpha n}(S) \hat{\Lambda}_{(n) A}^{+}\right) P(x, t) H_{\alpha}\right\}=0,
\end{array}
$$

where in the operators $\check{\Lambda}_{(n) A}^{+}$and $\hat{\Lambda}_{(n) A}^{+}$one must put $P^{\prime}(x, y, t)=P(x, y, s)$.

At fixed functions $\Omega_{\alpha}(\lambda, t)$ the one-parameter group of transformations (4.6) determines in an unexplicit form the flow $Y_{\Omega}: P(x, y, t) \rightarrow P\left(x, y, t^{\prime}\right)$, in other words, an evolution system. This evolution system can also be described by some nonlinear evolution equation.

Indeed let us consider the infinitesimal displacement in time : $t \rightarrow t^{\prime}=t+\varepsilon, \varepsilon \rightarrow 0$. In this case $P\left(x, y, t^{\prime}\right)=P(x, y, t)+\varepsilon \frac{\partial P(x, y, t)}{\partial t}$ and $B_{\alpha}(\lambda, t)=1-\varepsilon \Omega_{\alpha}(\lambda, t)$. Substituting these expressions into (4.6) and keeping the terms of the first order on $\varepsilon$, we obtain an evolution equation

$$
\frac{\partial P(x, y, t)}{\partial t}-\sum_{\alpha=1}^{r_{A}}\left(\Omega_{\alpha}\left(\check{L}_{A}^{+}, t\right) H_{\alpha} P-\Omega_{\alpha}\left(\hat{L}_{A}^{+}, t\right) P H_{\alpha}\right)=0
$$

where

$$
\left.\hat{L}_{(n) A}^{+} \stackrel{\text { def }}{=} \hat{\Lambda}_{(n) A}^{+}\right|_{P^{\prime}=P},\left.\quad \check{L}_{(n) A}^{+} \stackrel{\text { def }}{=} \check{\Lambda}_{(n) A}^{+}\right|_{P^{\prime}=P}
$$

and

$$
\begin{aligned}
& \Omega_{\alpha}\left(\hat{L}_{A}^{+} t\right) \stackrel{\text { def }}{=} \sum_{n=0}^{\infty} \omega_{\alpha n} \hat{L}_{(n) A}^{+}, \\
& \Omega_{\alpha}\left(\hat{L}_{A}^{+}, t\right) \stackrel{\text { def }}{=} \sum_{n=0}^{\infty} \omega_{\alpha n}(-1)^{n} \check{L}_{(n) A}^{+} .
\end{aligned}
$$

The operators $\hat{L}_{(n) A}^{+}$are calculated from the recursion relations (3.12) at $P^{\prime}=P$. For example

$$
\begin{aligned}
\hat{L}_{(1)}^{+} \cdot= & \frac{\partial}{\partial x}+A \frac{\partial}{\partial y}-[P, \cdot]_{F}-\left[P, \mathscr{I}^{+}[P, \cdot]_{0}\right], \\
\hat{L}_{(2) A}^{+} \Phi= & \left(\hat{L}_{(1) A}^{+}\right)^{2} \Phi-\left(\Phi_{A} P_{(1)}\right)_{F} \\
& -\mathscr{I}^{+}\left(\left[P, \Phi_{A}\right]\right)_{0} P_{(1)}-\left[P, \mathscr{I}^{+}\left(\Phi_{A} P_{(1)}\right)_{0}\right] .
\end{aligned}
$$

The operators $L_{(n) A}^{+}$can be calculated by the formula

$$
\check{L}_{(n) A}^{+}=(-1)^{n} \sum_{k=0}^{n} C_{k}^{n} \hat{L}_{(k) A}^{+} \frac{\partial^{n-k}}{\partial y^{n-k}} .
$$

1 Transformations of the form (4.6) were considered for the first time in [12] for a one-dimensional bundle (1.0) at $N=2, A=\left(\begin{array}{rr}1 & 0 \\ 0 & -1\end{array}\right)$ 
For the scattering matrix from (2.7) we correspondingly obtain the following linear evolution equation

$$
\frac{d \hat{S}(\tilde{\lambda}, \lambda, t)}{d t}=Y(\tilde{\lambda}, t) \hat{S}(\tilde{\lambda}, \lambda, t)-\hat{S}(\tilde{\lambda}, \lambda, t) Y(\lambda, t)
$$

where

$$
Y(\lambda, t) \stackrel{\text { def }}{=} \sum_{\alpha=1}^{r_{A}} \Omega_{\alpha}(\lambda, t) H_{\alpha} .
$$

The nonlinear evolution equation (4.7) determines the flow $Y_{\Omega}: P(x, y, t) \rightarrow P\left(x, y, t^{\prime}\right)$ in the infinitesimal form. The relation (4.6) which does not contain the derivative $\frac{\partial P}{\partial t}$ is an "integrated" form of the evolution equation (4.7). A class of Eq. (4.7) is characterized by an integer $N$, operators $\hat{L}_{(n) A}^{+} \breve{L}_{(n) A}^{+}$and arbitrary functions $\Omega_{1}(\lambda, t), \ldots, \Omega_{r_{A}}(\lambda, t)$, entire on $\lambda$. Let us point out that the evolution law of the scattering matrix of the type (4.10) was first considered by Zakharov [15].

The transformations (4.3) with matrices $B(\lambda, t)$ commuting with matrix $Y$ given by (4.11) (i.e. for $\left.B \subset g_{0(Y)}\right)$ form an infinite-dimensional group of Bäcklundtransformations for Eq. (4.7). At $\frac{\partial B_{\alpha}}{\partial t}=0$ the transformation (4.3), as it follows from (2.7), does not change the evolution law (4.10) of the scattering matrix and therefore they are auto-Bäcklund-transformations for Eq. (4.7): they transform solutions of an equation of the form (4.7) into solutions of the same equation. Some concrete auto-Bäcklund-transformations which have been found by other methods in $[20,21]$ are particular cases of the general transformations (4.3). If $\frac{\partial B_{\alpha}}{\partial t} \neq 0$, then the transformations (4.3) are generalized Bäcklund-transformations. Group $B$ of the transformation (4.3) also contains as a subgroup an infinitedimensional symmetry group of Eq. (4.7). In the infinitesimal form these symmetry transformations for the regular matrix $A$ are

$$
\begin{gathered}
P \rightarrow P^{\prime}=P+\delta P: \\
\delta P(x, y, t)=\sum_{\alpha=1}^{N}\left(f_{\alpha}\left(\check{L}_{A}^{+}\right) H_{\alpha} P-f_{\alpha}\left(\hat{L}_{A}^{+}\right) P H_{\alpha}\right),
\end{gathered}
$$

where $f_{\alpha}(\lambda)$ are arbitrary entire functions. Let us point out that the group of Bäcklund-transformations (4.3) and the symmetry group are universal ones, i.e. they are a group of Bäcklund-transformations and the symmetry group for all equations of the form (4.7).

One can also obtain Eq. (4.7) without using the transformation (4.3). Indeed, let the transformation $T: P \rightarrow P^{\prime}, S \rightarrow S^{\prime}$ be the infinitesimal displacement in time $t$ : $P^{\prime}=P+\varepsilon \frac{\partial P}{\partial t}, S^{\prime}=S+\varepsilon \frac{d S}{d t}, \varepsilon \rightarrow 0$. From the relation (2.6) we obtain

$$
\frac{d \hat{S}(\tilde{\lambda}, \lambda, t)}{d t}=-\int_{-i \infty}^{+i \infty} d \mu \hat{S}(\tilde{\lambda}, \mu) \int_{-\infty}^{+\infty} d x d y \breve{F}_{\mu}^{+}(x, y) \cdot \frac{\partial P(x, y, t)}{\partial t} \hat{F}_{\lambda}^{+}(x, y) .
$$


Using (4.12) and the identity (2.8) one can show that

$$
\begin{aligned}
& \frac{d \hat{S}(\tilde{\lambda}, \lambda, t)}{d t}-(Y(\tilde{\lambda}, t) \hat{S}(\tilde{\lambda}, \lambda, t)-\hat{S}(\tilde{\lambda}, \lambda, t) Y(\lambda, t)) \\
& =\int_{-i \infty}^{+i \infty} d \mu \hat{S}(\tilde{\lambda}, \mu, t) \int_{-\infty}^{+\infty} d x d y \check{F}_{\mu}^{+}(x, y)\left(\frac{\partial P(x, y, t)}{\partial t} \hat{F}_{\lambda}^{+}(x, y)\right. \\
& \left.\quad-Y\left(\frac{\partial}{\partial y}, t\right) P(x, y, t) \hat{F}_{\lambda}^{+}(x, y)+P(x, y, t) Y\left(\frac{\partial}{\partial y}, t\right) \hat{F}_{\lambda}^{+}(x, y)\right),
\end{aligned}
$$

where $Y(\lambda, t)$ is a matrix commuting with the matrix $A\left[\right.$ i.e. $Y=Y_{0}, Y(\lambda, t)$ $\left.=\sum_{\alpha=1}^{r_{A}} H_{\alpha} \sum_{n=0}^{\infty} \omega_{\alpha n}(t) \lambda^{n}\right]$. Then taking into account the relations (3.6) and (3.8) (at $P^{\prime}=P$ ) from (4.13), we obtain

$$
\begin{gathered}
\int_{-i \infty}^{+i \infty} d \mu\left\{\check{S}(\tilde{\lambda}, \mu, t)\left\{\frac{d \hat{S}(\mu, \lambda, t)}{d t}-(Y(\mu, t) \hat{S}(\mu, \lambda, t)-\hat{S}(\mu, \lambda, t) Y(\lambda, t)\}\right\}_{F}\right. \\
=-\int_{-\infty}^{+\infty} d x d y \operatorname{tr}\left\{\Phi_{(0) F}^{(F)}(x, y ; \tilde{\lambda}, \lambda)\right. \\
\left.\cdot\left(\frac{\partial P(x, y, t)}{\partial t}-\sum_{\alpha=1}^{r_{A}}\left(\Omega_{\alpha}\left(\check{L}_{A}^{+}, t\right) H_{\alpha} P-\Omega_{\alpha}\left(\hat{L}_{A}^{+}, t\right) P H_{\alpha}\right)\right)\right\} .
\end{gathered}
$$

From the equality (4.14) a connection between Eqs. (4.7) and (4.10) follows. In particular, if the scattering matrix $\hat{S}(\tilde{\lambda}, \lambda, t)$ satisfies Eq. (4.10), then potential $P(x, y, t)$ satisfies the evolution equation (4.7) (if $\Phi_{(0) F}^{(F)}$ form a complete set).

Let us also note that the transformation (4.3) and Eqs. (4.7) can be written in a form containing only one of the operators $\hat{\Lambda}_{(n) A}^{+}, \breve{\Lambda}_{(n) A}^{+}$. For example, in the form

$$
\sum_{\alpha=1}^{r_{A}} \sum_{n=0}^{\infty} b_{\alpha n}(t)\left(\sum_{m=0}^{n} C_{m}^{n} \hat{\Lambda}_{(m) A}^{+} H_{\alpha} P_{(n-m)}^{\prime}-\hat{\Lambda}_{(n) A}^{+} P H_{\alpha}\right)=0
$$

and

$$
\frac{\partial P(x, y, t)}{\partial t}-\sum_{\alpha=1}^{r_{A}} \sum_{n=0}^{\infty} \omega_{\alpha n}(t)\left(\sum_{m=0}^{n} C_{m}^{n} \hat{L}_{(m) A}^{+} H_{\alpha} P_{(n-m)}-\hat{L}_{(n) A}^{+} P H_{\alpha}\right)=0 .
$$

Equations (4.7) (or (4.16)) are just the nonlinear evolution equations in $1+2$ dimensions (one time and two spatial dimensions) integrable by the IST method with the help of the linear problem (1.1). Using the two-dimensional version of the IST method (see e.g. $[1,13,15]$ ) one can find, in principle, a broad class of exact solutions of Eqs. (4.7).

The class of Eqs. (4.7) contains some well-known nonlinear evolution equations in $1+2$ dimensions. For example, for a diagonal matrix $A\left(A_{i k}=a_{i} \delta_{i k}, a_{i} \neq a_{k}, i\right.$, $k=1, \ldots, N)$ and $Y_{i k}(\lambda)=\lambda \omega_{i} \delta_{i k}, i, k=1, \ldots, N$, where $\omega_{i}$ are some constants, Eq. (4.7) is

$$
\begin{aligned}
& \frac{\partial P_{i k}(x, y, t)}{\partial t}+\frac{\omega_{i}-\omega_{k}}{a_{i}-a_{k}} \frac{\partial P_{i k}}{\partial x}+\frac{a_{k} \omega_{i}-a_{i} \omega_{k}}{a_{i}-a_{k}} \frac{\partial P_{i k}}{\partial y} \\
& \quad+\sum_{l=1}^{N}\left(\frac{\omega_{i}-\omega_{l}}{a_{i}-a_{l}}-\frac{\omega_{l}-\omega_{k}}{a_{l}-a_{k}}\right) P_{i l} P_{l k}=0, \quad(i, k=1, \ldots, N) .
\end{aligned}
$$


The system of Eqs. (4.17) describing the two-dimensional resonantly interacting waves has been studied (for $N=3$ ) in Refs. $[13,14,17]$.

As the second example, we consider the case

$$
A=\left(\begin{array}{ll}
I_{N} & 0 \\
0-I_{M}
\end{array}\right), \quad Y(\lambda)=2 i \lambda^{2} A, \quad P=\left(\begin{array}{ll}
0 & Q \\
R & 0
\end{array}\right),
$$

where $I_{N}$ and $I_{M}$ are identical square matrices of the orders $N$ and $M$ respectively; $Q$ is a $N \times M$ rectangular matrix and $R$ is an $M \times N$ rectangular matrix. In this case Eq. (4.7) is the following system of matrix equations

$$
\begin{gathered}
i \frac{\partial Q}{\partial t}+\left(\frac{\partial^{2}}{\partial x^{2}}+\frac{\partial^{2}}{\partial y^{2}}\right) Q-Q V_{2}-V_{1} Q=0, \\
i \frac{\partial R}{\partial t}-\left(\frac{\partial^{2}}{\partial x^{2}}+\frac{\partial^{2}}{\partial y^{2}}\right) R+R V_{1}+V_{2} R=0, \\
V_{1}(x, y)=\int_{-\infty}^{x} d z\left((Q R)^{\prime}-(Q R)^{\prime \prime}\right)(z, z-x+y), \\
V_{2}(x, y)=\int_{-\infty}^{x} d z\left((R Q)^{\prime}-(R Q)^{\prime \prime}\right)(z, x-z+y),
\end{gathered}
$$

where $f^{\prime}(x, y) \stackrel{\text { def }}{=} \frac{\partial f(x, y)}{\partial x}, f^{\prime \prime}(x, y) \stackrel{\text { def }}{=} \frac{\partial f(x, y)}{\partial y}$. For $N=M=1$ the system of Eqs. (4.19) has been considered first in Refs. $[14,15]$.

For $M=1$, arbitrary $N$ and $R=Q^{+}$, system (4.19) reduces to a $1+2$ dimensional generalization of the $N$-component nonlinear Schroedinger equation

$$
\begin{aligned}
{\left[Q=\left(\begin{array}{c}
q_{1} \\
\vdots \\
q_{N}
\end{array}\right)\right] } & \\
i \frac{\partial q_{k}}{\partial t}+\left(\frac{\partial^{2}}{\partial x^{2}}+\frac{\partial^{2}}{\partial y^{2}}\right) & q_{k}-\sum_{n=1}^{N}\left(\delta_{k n} V_{(2)}+V_{(1) k n}\right) q_{n}=0, \quad(k=1, \ldots, N), \\
V_{(1) k n}(x, y) & =\int_{-\infty}^{x} d z\left(\left(q_{k} q_{n}^{*}\right)^{\prime}-\left(q_{k} q_{n}^{*}\right)^{\prime \prime}\right)(z, z-x+y), \\
V_{(2)}(x, y) & =\int_{-\infty}^{x} d z \sum_{l=1}^{N}\left(\left(q_{l} q_{l}^{*}\right)^{\prime}-\left(q_{l} q_{l}^{*}\right)^{\prime \prime}\right)(z, x-z+y) .
\end{aligned}
$$

For $N=1$, see $[14,15]$.

Let us note that in contrast to $1+1$ dimensional differential equations integrable by the problem (1.0), Eqs. (4.7) are integro-differential ones as a rule. The integro-differential equations (4.19), (4.20) can be also rewritten in the form of the systems of differential equations.

It is also interesting to consider the stationary equations (4.7), i.e. Eqs. (4.7) with $\frac{\partial P}{\partial t}=0$. These equations

$$
\sum_{\alpha=1}^{r_{A}}\left(\Omega_{\alpha}\left(\check{L}_{A}^{+}, t\right) H_{\alpha} P-\Omega_{\alpha}\left(\hat{L}_{A}^{+}, t\right) P H_{\alpha}\right)=0
$$


are the two-dimensional equations which contain the independent variables $x$ and $y$, putting them on more equal footing than $1+1$ dimensional equations $[$ e.g. Eqs. (4.7) at $\left.\frac{\partial P}{\partial y}=0\right]$ which contain the variables $t$ and $x$. For example, for $N=M=1, Q=R=U(x, y)$, the stationary equation (4.19) is equivalent to the following two-dimensional system of equations for two scalar fields $U(x, y)$ and $\varphi(x, y)$

$$
\begin{aligned}
\left(\frac{\partial^{2}}{\partial x^{2}}+\frac{\partial^{2}}{\partial y^{2}}\right) U & =U^{3}+U \varphi, \\
\left(\frac{\partial^{2}}{\partial x^{2}}-\frac{\partial^{2}}{\partial y^{2}}\right) \varphi & =\left(\frac{\partial}{\partial x}-\frac{\partial}{\partial y}\right)^{2} U^{2} .
\end{aligned}
$$

The system of nonstationary equations close to this system has been considered in $[1,18]$.

In conclusion let us consider the one-dimensional limit when the potential $P$ in (1.1) is independent of the variable $y\left(\frac{\partial P}{\partial y}=0\right)$. If one performs the Fourier transform of $\Psi(x, y, t)$ over variable $y$, i.e.

$$
\Psi(x, y, t)=(2 \pi i)^{-1 / 2} \int d \lambda \exp (\lambda y) \tilde{F}(x, \lambda, t)
$$

then the problem (1.1) is reduced to the one-dimensional bundle (1.0) for $\tilde{F}(x, \lambda, t)$. In this one-dimensional case

$$
\begin{aligned}
& \hat{F}_{\lambda}^{ \pm}(x, y, t)=(2 \pi i)^{-1 / 2} \exp (\lambda y) \hat{\tilde{F}}^{ \pm}(x, \lambda, t), \\
& \check{F}_{\lambda}^{ \pm}(x, y, t)=(2 \pi i)^{-1 / 2} \exp (-\lambda y) \check{\tilde{F}}^{ \pm}(x, \lambda, t),
\end{aligned}
$$

and from relations $(2.2)-(2.4)$ it follows that $\check{\tilde{F}}^{ \pm}=\left(\hat{\tilde{F}}^{ \pm}\right)^{-1}$, and

$$
\hat{S}(\tilde{\lambda}, \lambda, t)=\delta(\tilde{\lambda}-\lambda) S(\lambda, t), \quad \check{S}(\tilde{\lambda}, \lambda, t)=\delta(\tilde{\lambda}-\lambda) S^{-1}(\lambda, t)
$$

where $S(\lambda, t)$ is the one-dimensional scattering matrix.

Then for the expressions $\hat{\Phi}_{(n)}$ and $\breve{\Phi}_{(n)}$, we have

$$
\hat{\Phi}_{(n)}=\lambda^{n} \tilde{\Phi}(x, \lambda, t), \quad \check{\Phi}_{(n)}=(-\lambda)^{n} \tilde{\Phi}(x, \lambda, t)
$$

where

$$
\tilde{\Phi}(x, \lambda, t)=\hat{\tilde{F}}^{+}(x, \lambda, t) \otimes\left(\hat{\tilde{F}}^{+}(x, \lambda, t)\right)^{-1},
$$

and a symbol $\otimes$ denotes a tensor product of matrices. Furthermore, in the case $\frac{\partial P}{\partial y}=0$, all the terms in (3.7), (3.9) and (3.12) contained in the figured brackets are equal to zero and therefore relations (3.6)-(3.14) are reduced to the following one:

$$
\lambda \tilde{\Phi}_{F}(x, \lambda, t)=\hat{\Lambda}_{(1) A} \tilde{\Phi}_{F}(x, \lambda, t)
$$


and

$$
\begin{gathered}
\hat{\Lambda}_{(n) A}=\left(\hat{\Lambda}_{(1) A}\right)^{n}, \quad \hat{\Lambda}_{(n) A}^{+}=\left(\hat{\Lambda}_{(1) A}^{+}\right)^{n}, \\
\check{\Lambda}_{(n) A}=\left(-\hat{\Lambda}_{(1) A}\right)^{n}, \quad \check{\Lambda}_{(n) A}^{+}=\left(-\hat{\Lambda}_{(1) A}^{+}\right)^{n}, \quad(n=1,2,3, \ldots) .
\end{gathered}
$$

In the result for $\frac{\partial P}{\partial y}=0$, the transformations (4.3) and Eqs. (4.7) are reduced to the corresponding transformations and equations connected to the onedimensional bundle (1.0) (see Refs. [7-9]).

Let us also emphasize that in contrast to the one-dimensional case (1.0) [see (4.21)], for the two-dimensional problem (1.1), the recursion operators $\Lambda_{(n) A}$ and $\Lambda_{(n) A}^{+}$are not the powers of the operators $\Lambda_{(1) A}$ and $\Lambda_{(1) A}^{+}[$see $(3.7)-(3.14)]$.

\section{Integrals of Motion}

Here for simplicity we consider the case of a regular matrix $A$. In this case the subalgebra $g_{0}$ is abelian one.

Let us note that in view of $(4.10)$ the quantity $S_{0}(\lambda, \lambda)$ is time-independent:

$$
\frac{d \hat{S}_{0}(\lambda, \lambda)}{d t}=0
$$

for any functions $\Omega_{\alpha}(\lambda, t)$. Therefore $\hat{S}_{0}(\lambda, \lambda)$ are integrals of motion for any $\lambda$. Expanding (analogous to the $1+1$ dimensional case $[1,2]$ ) the quantity $\ln S_{0}(\lambda, \lambda)$ in the asymptotic series on $\lambda^{-1}: \ln \hat{S}_{0}(\lambda, \lambda)=\sum_{n=1}^{\infty} \lambda^{-n} C^{(n)}$, we obtain a countable set of integrals of motion $C^{(n)}(n=1,2, \ldots)$ for Eqs. (4.7). Analogous to the $1+1$ dimensional case, the integrals of motion $C^{(n)}$ can be written as functionals over the potential $P(x, y, t)$. Let us represent for this purpose the matrix-solution $\hat{F}_{\kappa}^{+}(x, y, t)$ in the form

$$
\hat{F}_{\lambda}^{+}(x, y, t)=R_{\lambda}(x, y, t) E(x, y) \exp \left(\chi_{\lambda}(x, y, t)\right)
$$

where $E_{\lambda}(x, y)=(2 \pi i)^{-1 / 2} \exp \lambda(y-A x),\left(\chi_{\lambda}\right)_{0}=\chi_{\lambda}$ and $\left(R_{\lambda}\right)_{0}=1$. Passing in (5.2) to the limit $x \rightarrow-\infty$ and taking the projection on the subspace $g_{0}$, we obtain

$$
\ln \hat{S}_{0}(\lambda, \lambda)=\ln \left(\int_{-\infty}^{+\infty} d y \exp \left(\chi_{\lambda}(-\infty, y)\right)\right.
$$

Thus the integrals of motion $C^{(n)}$ are the coefficients in the asymptotic expansion on $\lambda^{-1}$ of the right-hand side of equality (5.3). These coefficients are connected in an obvious way with the coefficients $\chi_{(n)}(y)$ of the asymptotic expansion on $\lambda^{-1}$ of the quantity $\chi_{\lambda}(-\infty, y)$

$$
\chi_{\lambda}(-\infty, y)=\sum_{n=1}^{\infty} \lambda^{-n} \chi_{(n)}(y) \text {. }
$$


As a result

$$
\begin{aligned}
& C^{(1)}=\int_{-\infty}^{+\infty} d y \chi_{(1)}(y), \\
& C^{(2)}=\int_{-\infty}^{+\infty} d y\left(\chi_{(2)}(y)+\frac{1}{2} \chi_{(1)}^{2}(y)\right)-\frac{1}{2}\left(\int_{-\infty}^{+\infty} d y \chi_{(1)}(y)\right)^{2},
\end{aligned}
$$

and so on.

The expressions for $\chi_{(n)}(y)$ are found from the recursion relations which, analogous to the $1+1$ dimensional case, are obtained by substituting $\hat{F}_{\lambda}^{+}(x, y)$ in the form (5.2) into the linear problem (1.1). They are of the form

$$
\chi_{(n)}(y)=-\mathscr{I}^{-}\left(P R^{(n)}\right)_{0}, \quad(n=1,2, \ldots),
$$

where $R^{(n)}$ are calculated by the recursion relations

$$
\begin{aligned}
&\left(R_{\lambda}(x, y)=1+\sum_{n=1}^{\infty} \lambda^{-n} R^{(n)}(x, y)\right) \\
& R^{(1)}=P_{A}, \\
& R^{(n+1)=}-\left(\frac{\partial}{\partial x}+A \frac{\partial}{\partial y}\right) R_{A}^{(n)}-\sum_{q=1}^{n-1} R_{A}^{(q)} \frac{\partial}{\partial x} \mathscr{I}^{-}\left(P R^{(n-q)}\right)_{0} \\
&-A \sum_{q=1}^{n-1} R_{A}^{(q)} \frac{\partial}{\partial y} \mathscr{I}^{-}\left(P R^{(n-q)}\right)_{0}+\left(P R^{(n)}\right)_{F}=0, \quad(n=1,2, \ldots) .
\end{aligned}
$$

Let us emphasize that the integrals of motion $C^{(n)}$ are universal. Indeed in their calculation we use only the time-independence of $S_{0}(\lambda, \lambda)$ and the spectral problem (1.1) but not Eqs. (4.7). Therefore $C^{(n)}$ are integrals of motion for any equations of the form (4.7).

In the particular case of a diagonal regular matrix $A$ and $N=3$, the procedure for calculation of the integrals of motion in $1+2$ dimensions described in this section is close to those given earlier in $[17,18,22]$.

\section{Reduction Problem}

Similar to the $1+1$ dimensions case, the reduction problem for general equations (4.7). i.e. the problem of effective decreasing of the number of the independent fields in these equations is an important one.

In the $1+1$ dimensions case Mikhailov $[23,24]$ proposed a very interesting approach to the reduction problem. This approach is based on the introduction of the notion of the reduction group, in other words, the group of the forminvariance of the potential. In the framework of the AKNS-approach, the reduction problem also leads to the problem of the enumeration of those functions $\Omega_{\alpha}(\lambda, t)$ for which the integrable equations admit certain reduction. In the $1+1$ dimensional case and a bundle (1.0) this problem was solved in [25]. A related approach for another spectral problem was proposed in [26, 27$]$. 
Here we consider the reduction problem for Eqs. (4.7) integrable by the problem (1.1). Let us consider for definiteness $Z_{N}$ reduction. (For the $1+1$ dimensional case, see [23-25].) The $Z_{N}$ reduction is generated by the constraints

$$
G A=q A G, \quad G P(x, y, t)=P(x, y, t) G,
$$

where $G_{i k}=\delta_{i, k-1}(i, k=1, \ldots, N), G_{N 1}=1$ and $q=\exp \frac{2 \pi i}{N}$. Under $Z_{N}$ reduction the potential $P$ has only $N-1$ independent variables and $A_{i k}=q^{i-1} \delta_{i k}$ [23-25]. Let $\hat{\Psi}_{\lambda}(x, y)$ be a solution to the problem (1.1). Let us consider the expression $\Psi^{\prime}(x, y)$ $=G \hat{\Psi}_{\lambda}(x, y)$. As $|x| \rightarrow \infty$, it satisfies the equation $\frac{\partial \Psi^{\prime}}{\partial x}+q A \frac{\partial \Psi^{\prime}}{\partial y}=0$ and therefore in the limit $|x| \rightarrow \infty$ it can be represented as follows

$$
G \hat{\Psi}_{\lambda}(x, y)=\int_{-i \infty}^{+i \infty} d \mu \hat{\Psi}_{\mu}\left(x, q^{-1} y\right) T(\mu, \lambda)
$$

where $T(\mu, \lambda)$ is a matrix. Using (2.2) and (6.2), we obtain

$$
T(\mu, \lambda)=\left.\int_{-\infty}^{+\infty} d y \check{\Psi}_{\mu}(x, y) G \hat{\Psi}_{\lambda}(x, q y)\right|_{|x| \rightarrow \infty} .
$$

Setting for example $\hat{\Psi}_{\lambda}=\hat{F}_{\lambda}^{+}$and $x \rightarrow+\infty$, one gets

$$
T(\mu, \lambda)=\delta(\mu-q \lambda) G
$$

As a result, the relation (6.2) takes the form

$$
\left.G \hat{\Psi}_{\lambda}(x, y)\right|_{|x| \rightarrow \infty}=\left.\hat{\Psi}_{q \lambda}\left(x, q^{-1} y\right) G\right|_{|x| \rightarrow \infty} .
$$

Furthermore, since the relation (6.2) is valid at $|x| \rightarrow \infty$ both for $F^{+}$and $F^{-}$, we obtain the following equation for the scattering matrix

$$
\int_{-i \infty}^{+i \infty} d \mu \hat{S}(\tilde{\lambda}, \mu) T(\mu, \lambda)=\int_{-i \infty}^{+i \infty} d \mu T(\tilde{\lambda}, \mu) S(\mu, \lambda)
$$

Taking into account (6.4), we have

$$
G \hat{S}(\tilde{\lambda}, \lambda, t) G^{-1}=\hat{S}(q \tilde{\lambda}, q \lambda, t) .
$$

Furthermore, demanding the consistency of the constraint (6.7) with Eq. (4.10), we obtain

$$
G Y(\lambda, t) G^{-1}=Y(q \lambda, t)
$$

Since $Y(\lambda, t)=\sum_{\alpha=1}^{r_{A}} \Omega_{\alpha}(\lambda, t) H_{\alpha}$, we find from (6.8) that $\Omega_{\alpha}(\lambda, \mathrm{t})=\Omega_{1}\left(q^{\alpha-1} \lambda, t\right)$ $(\alpha=1, \ldots, N)$, i.e. $\Omega_{\alpha}(\lambda, t)=\sum_{n=1}^{N-1} q^{(\alpha-1) n} \lambda^{n} \Omega_{n}\left(\lambda^{N}, t\right)$, where $\Omega_{n}\left(\lambda^{N}, t\right)$ are arbitrary functions entire on $\lambda^{N}$. As a result,

$$
Y(\lambda, t)=\sum_{n=1}^{N-1} \lambda^{n} \Omega_{n}\left(\lambda^{N}, t\right) A^{n} .
$$


The expression (6.9) gives us the general form of $Y(\lambda, t)$ for which Eqs. (4.7) admit a nontrivial $Z_{N}$-reduction (6.1). Indeed, for such $Y(\lambda, t)$ the relation (6.7) is consistent with Eq. (4.10) and, as a result, as it follows from the equality (4.14), the constraint (6.1) is consistent with Eq. (4.7).

In the general case dealing with the reduction problem one can act in the same way as in the example considered above:

1) first, we find the reduction group, i.e. we find the constraints on a matrix $A$ and a potential $P$,

2) calculate matrix $T(\mu, \lambda)$ using $(6.3)$,

3) obtain constraints for $Y(\lambda, t)$ analogous to (6.8), and

4) solve this constraint and find the general form of the functions $\Omega_{\alpha}(\lambda, t)$ for which Eqs. (4.7) admit the given reduction.

Let us consider as an illustration some other reductions. The general equation (4.7) admit the reduction $P^{T}(x, y, t)=-P(x, y, t)$ at $A^{T}=A$ and arbitrary odd functions $\Omega_{\alpha}(\lambda, t)$.

Similar to the $1+1$ dimensional case [28], the so-called $\tilde{Z}_{N}$ reduction is important. This is the reduction

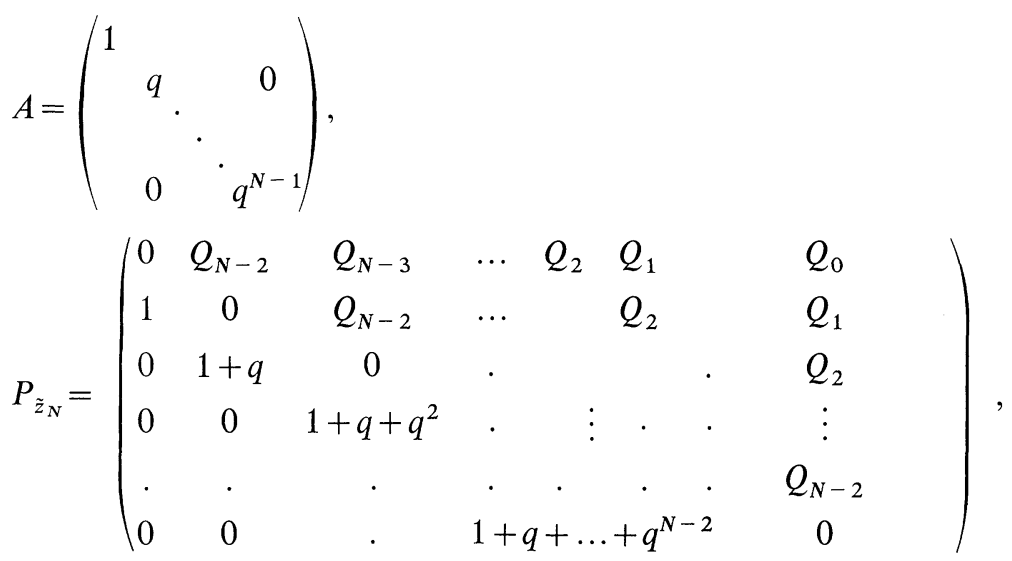

where $q=\exp \frac{2 \pi i}{N}$. One can show that Eqs. (4.7) admit $\tilde{Z}_{N}$ reduction $(6.10)$ at $Y(\lambda, t)$ $=\sum_{n=1}^{N-1} \lambda^{n} \Omega_{n}\left(\lambda^{N}, t\right) A^{n}$, i.e. for the same class of functions $\Omega_{\alpha}$ as for $Z_{N}$ reduction (6.1). It is not difficult to prove that the linear problem (1.1) under $\tilde{Z}_{N}$ reduction is equivalent to the following two-dimensional problem $\left[\tilde{\Psi}^{T}=\left(\Psi_{1}, \ldots, \Psi_{N}\right)\right]$

$$
\sum_{k=0}^{N} V_{k}(x, y) \frac{\partial^{k}}{\partial x^{k}} \Psi_{N}-\frac{\partial^{N}}{\partial y^{N}} \Psi_{N}=0
$$

where $V_{N}=1, V_{N-1}=0$. The coefficients $V_{0}(x, y), \ldots, V_{N-2}(x, y)$ are simply expressed through $Q_{0}, \ldots, Q_{N-2}$. For example, for $N=2(q=-1), V_{0}(x, y)=-Q_{0}(x, y)$. The corresponding spectral problem is

$$
\left(\frac{\partial^{2}}{\partial x^{2}}-\frac{\partial^{2}}{\partial y^{2}}\right) \Psi_{2}+V_{0}(x, y) \Psi_{2}=0
$$


and it has been considered in Ref. [29]. For $N=3\left(q=\exp \frac{2 \pi i}{3}\right)$

$$
V_{0}=-(1+q)\left(\frac{\partial Q_{1}}{\partial x}+\frac{\partial Q_{1}}{\partial y}+Q_{0}\right), \quad V_{1}=-(2+q) Q_{1}
$$

or

$$
Q_{0}=-\frac{1}{1+q} V_{0}+\frac{1}{2+q}\left(\frac{\partial}{\partial x}+\frac{\partial}{\partial y}\right) V_{1}, \quad Q_{1}=-\frac{1}{2+q} V_{1} .
$$

The spectral problem (6.11) is one of possible two-dimensional generalizations of the well-known Gelfand-Dickey spectral problem

$$
\sum_{k=0}^{N} V_{k}(x) \frac{\partial^{k}}{\partial x^{k}} \chi-\lambda^{N} \chi=0
$$

Another two-dimensional generalization of the Gelfand-Dickey problem is

$$
\sum_{k=0}^{N} V_{k}(x, y) \frac{\partial^{k}}{\partial x^{k}} \chi+\alpha \frac{\partial \chi}{\partial y}=0
$$

and it can be obtained as a special reduction of the problem (1.1) with a degenerated matrix $A$. For example, the second order problem

$$
\frac{\partial \Psi}{\partial x}+\left(\begin{array}{ll}
0 & \alpha \\
0 & 0
\end{array}\right) \frac{\partial \Psi}{\partial y}=\left(\begin{array}{cc}
0 & -U \\
1 & 0
\end{array}\right) \Psi
$$

is equivalent to the scalar problem

$$
\alpha \frac{\partial \Psi_{2}}{\partial y}+\frac{\partial^{2} \Psi_{2}}{\partial x^{2}}+U(x, y, t) \Psi_{2}=0,
$$

which is used for the integration of the Kadomtsev-Petviashvili equation $[13,30]$.

Let us consider the families of Eqs. (4.7) under $Z_{2}$ and $\tilde{Z}_{2}$ reductions $(N=2)$. It follows from the obtained results that Eqs. (4.7) admit the reductions at $A=\left(\begin{array}{rr}1 & 0 \\ 0 & -1\end{array}\right)$ and $Y=\lambda \Omega\left(\lambda^{2}\right) A$, where $\Omega\left(\lambda^{2}\right)$ is an arbitrary entire function on $\lambda^{2}$. For $Z_{2}$ reduction $P=\left(\begin{array}{ll}0 & q \\ q & 0\end{array}\right)$, for the $\tilde{Z}_{2}$ reduction $P=\left(\begin{array}{ll}0 & q \\ 1 & 0\end{array}\right)$. For $\Omega\left(\lambda^{2}\right)=-2^{2 n}\left(\lambda^{2}\right)^{n},(n=1,2, \ldots)$, and the $\tilde{Z}_{2}$ reduction a family of Eqs. $(4.7)(N=2)$ is a generalization of the well-known KdV-family to $1+2$ dimensions. The simplest $(n=1)$ equation among these equations is a $1+2$ dimensions generalization of the KdV-equation considered in Ref. [29]. At $\Omega\left(\lambda^{2}\right)=-2^{2 n}\left(\lambda^{2}\right)^{n}(n=1,2, \ldots)$ and the $Z_{2}$-reduction the family of equations $(4.7)(N=2)$ is a $1+2$ dimensional generalization of the modified KdV-family. The simplest $(n=1)$ of these equations is a generalization of $\mathrm{mKdV}$-equation on $1+2$ dimensions.

In conclusion let us make the following important remark. All the formulas which we have used above in the analysis of the reductions contain only asymptotics of the solutions of the problem (1.1) as $|x| \rightarrow \infty$. We also do not demand that the problem (1.1) be invariant under the transformation 
$\hat{\Psi}_{\lambda}(x, y) \rightarrow \hat{\Psi}^{\prime}(x, y)=G \Psi_{\lambda}(x, y)$, i.e. we do not demand that $G \Psi_{\lambda}(x, y)$ be the solution of the problem (1.1). Here there is an important difference between the case of the two-dimensional problem (1.1) and the case of the one-dimensional bundle (1.0). While for the one-dimensional bundle the reduction group, i.e. the group of the form-invariance of the potential, is at the same time the symmetry group of the bundle (1.0), for the two-dimensional problem the reduction group [group of form-invariance of the potential $P(x, y, t)]$ is not a symmetry group of the problem (1.1).

Of course one may demand in the two-dimensional case that the reduction group will be the symmetry group of the problem (1.1) too. It is not difficult to see that in this case one must demand that the variable $y$ be transformed in a nontrivial way. For example, for the $Z_{N}$ reduction we must demand $y \rightarrow y^{\prime}=q^{-1} y$ and instead of $(6.1)$ the potential $P(x, y, t)$ should satisfy the constraint $G P(x, y, t) G^{-1}=P\left(x, q^{-1} y, t\right)$ for the whole range of the variables $x$ and $y$. Any reasonable interpretation of such constraints is not known.

\section{Conclusion}

The results of the present paper, analogous to $1+1$ dimensions [8,25], can be generalized to the problem (1.1) with $Z_{2}$-grading (the potential contains both commuting and anticommuting fields), to the case when $\lim _{R=\sqrt{x^{2}+y^{2}} \rightarrow \infty} P(x, y, t) \neq 0$, and to other spectral problems. Hamiltonian and group-theoretical structure of the evolution equations (4.7) will be considered elsewhere.

Acknowledgements. I am grateful to Prof. V. E. Zakharov, Drs. E. A. Kuznetsov, S. V. Manakov and A. V. Mikhailov for useful discussions of various aspects of the multidimensional IST method. I am indebted to Prof. D. J. Kaup who kindly sent me reprints and preprints of his papers on the threedimensional three-wave equations. I am also grateful to Prof. Ya. G. Sinai for numerous useful remarks.

\section{References}

1. Zakharov, V.E., Manakov, S.V., Novikov, S.P., Pitaevski, L.P.: Solition theory. The method of inverse problem. Moscow: Nauka 1980 (in Russian)

2. Solitons. Topics in Current Physics, Vol.17, Bullough, R., Caudrey, P. (eds.) Heidelberg: Springer 1980

3. Ablowitz, M.J., Kaup, D.J., Newell, A.C., Segur, H.: Stud. Appl. Math. 53, 249-315 (1974)

4. Miodek, I.: J. Math. Phys. 19, 19-31 (1978)

5. Newell, A.C.: Proc. R. Soc. (London) A365, 283-311 (1979)

6. Kulish, P.P. : Notes of LOMI scientific seminars 96, 105-112 (1980)

7. Konopelchenko, B.G.: Phys. Lett. 75 A, 447-450 (1980); preprint Institute of Nuclear Physics N 79-82 (1979)

8. Konopelchenko, B.G.: Phys. Lett. 79 A, 39-43 (1980); Phys. Lett. 95B, 83-89 (1980); Phys. Lett. 100B, 254-260 (1981)

9. Konopelchenko, B.G.: J. Phys. A: Math. Gen. 14, 1237-1259 (1981); 14, 3125-3141 (1981)

10. Calogero, F., Degasperis, A.: Nuovo Cimento 39B, 1-54 (1977)

11. Gerdjikov, V.S., Ivanov, M.I., Kulish, P.P.: Teor. Mat. Fyz. 44, 342-351 (1980) (in Russian)

12. Calogero, F., Degasperis, A.: Nuovo Cimento 32B, 201-243 (1976) 
13. Zakharov, V.E., Shabat, A.B.: Funct. Anal. Appl. 8, N3, 43-53 (1974); 13, N3, 13-22 (1979) (in Russian)

14. Ablowitz, M.J., Haberman, R. : Phys. Rev. Lett. 35, 1185-1188 (1975)

15. Zakharov, V.E.: The inverse scattering method. In: Solitons Ref. [2], pp. 243-285 (1980)

16. Zakharov, V.E., Manakov, S.V.: Sov. Phys. Rev. 1, 133 (1979)

17. Kaup, D.J.: Physica 1D, 45-67 (1980); Stud. Appl. Math. 62, 75-79 (1980)

18. Zakharov, V.E., Schulman, E.I.: Physica 1D, 192-202 (1980)

19. Bourbaki, N.: Groups et Algebras de Lie. Paris: Hermann 1972

20. Levi, D., Pilloni, L., Santini, P.M. : Phys. Lett. 81 A, 419 (1981)

21. Kaup, D.J.: J. Math. Phys. 22, 1176-1180 (1981)

22. Schulman, E.I.: Teor. Mat. Fyz. 44, 224 (1980) (in Russian)

23. Mikhailov, A.V.: Pisma v Zh. E.T.P. 30, 443 (1979); 32, 187 (1980) (in Russian)

24. Mikhailov, A.V.: In: Proc. of Soviet-American Symposium on Soliton Theory, September 1979, Kiev, Manakov, S.V., Zakharov, V.E. (eds.), Physica 3D, 73-117 (1981)

25. Konopelchenko, B.G.: Phys. Lett. 108 B, 26-32 (1982); preprint Institute of Nuclear Physics N81-99 (1981)

26. Calogero, F., Degasperis, A.: J. Math. Phys. 22, 23 (1981)

27. Degasperis, A.: In: Lecture Notes in Physics, Vol. 120, pp. 16-34. Berlin, Heidelberg, New York: Springer 1980

28. Konopelchenko, B.G.: Funct. Anal. Appl. 16, N3 (1982); preprint Institute of Nuclear Physics N80-223 (1980) (in Russian)

29. Nizhnik, L.P.: Dokl. Akad. Nauk SSSR 254, 332-335 (1980) (in Russian)

30. Dryuma, V.S. : JETP Lett. 19, 387-389 (1974)

Communicated by Ya. G. Sinai

Received December 5, 1981 
